\title{
Supermembrane limit of Yang-Mills theory
}

Olaf Lechtenfeld, and Alexander D. Popov

Citation: Journal of Mathematical Physics 57, 023520 (2016);

View online: https://doi.org/10.1063/1.4942186

View Table of Contents: http://aip.scitation.org/toc/jmp/57/2

Published by the American Institute of Physics

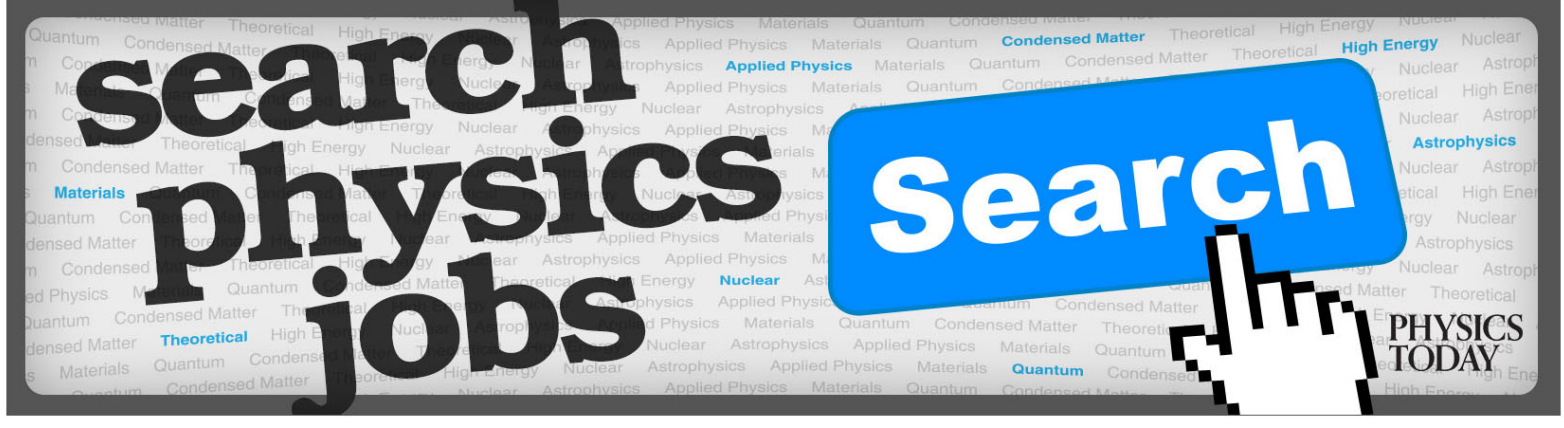




\title{
Supermembrane limit of Yang-Mills theory
}

\author{
Olaf Lechtenfeld ${ }^{\text {a) }}$ and Alexander D. Popova) \\ Institut für Theoretische Physik and Riemann Center for Geometry and Physics, Leibniz \\ Universität Hannover, Appelstraße 2, 30167 Hannover, Germany
}

(Received 27 November 2015; accepted 3 February 2016; published online 19 February 2016)

\begin{abstract}
We consider Yang-Mills theory with $N=1$ super-translation group in eleven auxiliary dimensions as the structure group. The gauge theory is defined on a direct product manifold $\Sigma_{3} \times S^{1}$, where $\Sigma_{3}$ is a three-dimensional Lorentzian manifold and $S^{1}$ is a circle. We show that in the infrared limit, when the metric on $S^{1}$ is scaled down, the Yang-Mills action supplemented by a Wess-Zumino-type term reduces to the action of an M2-brane. (C) 2016 AIP Publishing LLC. [http://dx.doi.org/10.1063/1.4942186]
\end{abstract}

\section{INTRODUCTION AND SUMMARY}

The theory of membranes and supermembranes has been developed for a long time. ${ }^{1-9,24}$ Supermembranes are basic objects (M2-branes) of M-theory, which are needed for constructing an effective theory of multi-M2-branes. ${ }^{9}$ In this paper, we show that the action of supermembranes moving in $d=11$ flat $N=1$ extended superspace can be obtained from a Yang-Mills action functional on $\Sigma_{3} \times S^{1}$ amended by a Wess-Zumino-type term when $S^{1}$ shrinks to a point.

Our construction is based on the adiabatic approach to differential equations (introducing "slow" and "fast" variables) which for a direct product manifold ${ }^{25} Z=X \times Y$ is equivalent to the introduction of a metric $g_{X}+\varepsilon^{2} g_{Y}$ with a real parameter $\varepsilon \in[0, \infty)$ and a consideration of the limit $\varepsilon \rightarrow 0 .{ }^{10,11,26}$ The adiabatic limit method has been applied to the description of the scattering of monopoles (i.e., constructing time-dependent solutions of the Yang-Mills-Higgs model), and it has been shown that in the limit $\varepsilon \rightarrow 0$, the scattering of monopoles is parametrized by geodesic motion on the moduli space $\mathcal{M}_{n}$ of $n$-monopoles. ${ }^{15,16}$ In other words, the Yang-Mills-Higgs system on $\mathbb{R}^{3,1}=\mathbb{R}^{0,1} \times \mathbb{R}^{3,0}$ for "slow time" reduces to a sigma model on $\mathbb{R}^{0,1}$ (time axis) with $\mathcal{M}_{n}$ as the target space.

In four dimensions, when $\operatorname{dim} Z=4$, one has $\operatorname{dim} X=1,2$, or 3 and $\operatorname{dim} Y=3,2$ or 1 , respectively. In Ref. 10, the adiabatic method was applied to the Yang-Mills instanton equations on a direct product $X \times Y$ of two Riemann surfaces, and it was shown that instanton solutions on $X \times Y$ are in a one-to-one correspondence with holomorphic maps from $X$ into the moduli space $\mathcal{M}$ of flat connections on $Y$. In this case, the Yang-Mills action reduces to the action of a sigma model on $X$ while $Y$ shrinks to a point. The sigma-model target space is $\mathcal{M}$, and holomorphic maps $X \rightarrow \mathcal{M}$ are the sigma-model instantons. The same result for the Lorentzian signature with $X=\mathbb{R}^{1,1}$ and $Y=T^{2}$ (two-torus) was derived in Ref. 12: Yang-Mills theory on $\mathbb{R}^{1,1} \times T^{2}$ in the infrared limit $\varepsilon \rightarrow 0$ (the size of $T^{2}$ tends to zero) reduces to a sigma model on $\mathbb{R}^{1,1}$ whose target space is the moduli space of flat connections on $T^{2}$. In Refs. 13 and 14, the same approach was applied to Yang-Mills theory ${ }^{27}$ on $\mathbb{R}^{2,1} \times S^{1}$. It was shown that Yang-Mills theory on $\mathbb{R}^{2,1} \times S^{1}$ reduces to a sigma model on $\mathbb{R}^{2,1}$ whose target space is the space of vacua that arise in the compactification on $S^{1}$. Finally, the adiabatic approach is natural and especially helpful in studying Yang-Mills instantons in more than four dimensions as it was shown in Refs. 11 and 17 (see also Ref. 18 and references therein).

To sum up, Yang-Mills theory on a manifold $X \times Y$ with metric $g_{X}+\varepsilon^{2} g_{Y}$ flows in the infrared limit $\varepsilon \rightarrow 0$ to a sigma model on $X$ whose target space is the moduli space $\mathcal{M}$ of flat connections on $Y$ when $\operatorname{dim} Y \leq 2$. In our short paper, we reverse this logic. For a given sigma model on $X$, we construct a Yang-Mills model on $X \times Y$ such that in the infrared limit $\varepsilon \rightarrow 0$, one gets back the

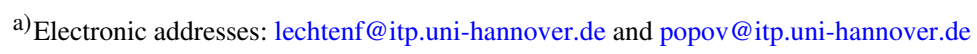


initial sigma model. In Refs. 19 and 20, this algorithm was carried out for the bosonic string and for the Green-Schwarz superstring in a $d=10$ Minkowski background. Here we apply this idea to the sigma model describing a supermembrane in a $d=11$ Minkowski background $^{5,7}$ and introduce a Yang-Mills model on $\Sigma_{3} \times S^{1}$ whose low-energy limit recovers the supermembrane action on $\Sigma_{3}$.

\section{LIE SUPERGROUP $G$}

We consider Yang-Mills theory on a direct product manifold $M^{4}=\Sigma_{3} \times S^{1}$, where $\Sigma_{3}$ is a three-dimensional Lorentzian manifold with local coordinates $x^{a}, a, b, \ldots=0,1,2$, and a metric tensor $g_{\Sigma_{3}}=\left(g_{a b}\right)$, and on the circle $S^{1}$ of unit radius parametrized by $x^{3} \in[0,2 \pi]$, we choose the metric $g_{S^{1}}=\left(g_{33}\right)$ with $g_{33}=1$. Then $\left(x^{\mu}\right)=\left(x^{a}, x^{3}\right)$ are local coordinates on $M^{4}$ with the metric tensor $\left(g_{\mu \nu}\right)=\left(g_{a b}, g_{33}\right), \mu, v, \ldots=0, \ldots, 3$. Having in mind open membranes, we assume that $\Sigma_{3}$ has a Lorentzian boundary $\Sigma_{2}=\partial \Sigma_{3}$. For closed membranes, $\Sigma_{2}$ is the empty set.

As the Yang-Mills structure group on $M^{4}$, we consider the coset $G=\operatorname{SUSY}(N=1) / \operatorname{SO}(10,1)$ (cf., Ref. 7), where $\operatorname{SUSY}(N=1)$ is the super-Poincare group in $d=11$ dimensions. The coset $G$ is the super-translation group in $d=11$ auxiliary dimensions. Its generators span the Lie superalgebra $\mathfrak{g}=$ Lie $G$,

$$
\left\{\xi_{A}, \xi_{B}\right\}=\left(\gamma^{\alpha} C\right)_{A B} \xi_{\alpha}, \quad\left[\xi_{\alpha}, \xi_{A}\right]=0, \quad\left[\xi_{\alpha}, \xi_{\beta}\right]=0,
$$

where $\gamma^{\alpha}$ are the gamma matrices in $d=11, C$ is the charge conjugation matrix, $\alpha=0, \ldots, 10$ and $A=1, \ldots, 32$. The coordinates on $G$ are denoted by $X^{\alpha}$ and by the components $\theta^{A}$ of a Majorana spinor $\theta=\left(\theta^{A}\right)$, whose conjugate is $\bar{\theta}=\theta^{\top} C$. The one-forms

$$
\Pi^{\Delta}=\left\{\Pi^{\alpha}, \Pi^{A}\right\}=\left\{\mathrm{d} X^{\alpha}-\mathrm{i} \bar{\theta} \gamma^{\alpha} \theta, \mathrm{d} \theta^{A}\right\}
$$

form a basis of (left-invariant) one-forms on $G^{5,7}$ On the superalgebra $\mathfrak{g}=$ Lie $G$, we introduce the scalar product $\langle\cdot \cdot\rangle$ such that

$$
\left\langle\xi_{\alpha} \xi_{\beta}\right\rangle=\eta_{\alpha \beta}, \quad\left\langle\xi_{\alpha} \xi_{A}\right\rangle=0 \text { and }\left\langle\xi_{A} \xi_{B}\right\rangle=0,
$$

where $\left(\eta_{\alpha \beta}\right)=\operatorname{diag}(-1,1, \ldots, 1)$ is the Lorentzian metric on $\mathbb{R}^{10,1}$.

\section{ACTION FUNCTIONAL}

Let us consider the gauge potential $\mathcal{A}=\mathcal{A}_{\mu} \mathrm{d} x^{\mu}$ with values in $\mathfrak{g}$ and the $\mathfrak{g}$-valued gauge field

$$
\mathcal{F}=\frac{1}{2} \mathcal{F}_{\mu \nu} \mathrm{d} x^{\mu} \wedge \mathrm{d} x^{\nu} \quad \text { with } \quad \mathcal{F}_{\mu \nu}=\partial_{\mu} \mathcal{A}_{\nu}-\partial_{\nu} \mathcal{A}_{\mu}+\left[\mathcal{A}_{\mu}, \mathcal{A}_{\nu}\right],
$$

where $[\cdot, \cdot]$ is the commutator or anti-commutator depending on the Grassmann parity of its arguments. On $\Sigma_{3} \times S^{1}$ we have the obvious splitting

$$
\begin{gathered}
\mathrm{d} s^{2}=g_{\mu \nu} \mathrm{d} x^{\mu} \mathrm{d} x^{\nu}=g_{a b} \mathrm{~d} x^{a} \mathrm{~d} x^{b}+\left(\mathrm{d} x^{3}\right)^{2}, \\
\mathcal{A}=\mathcal{A}_{\mu} \mathrm{d} x^{\mu}=\mathcal{A}_{a} \mathrm{~d} x^{a}+\mathcal{A}_{3} \mathrm{~d} x^{3}, \\
\mathcal{F}=\frac{1}{2} \mathcal{F}_{\mu \nu} \mathrm{d} x^{\mu} \wedge \mathrm{d} x^{\nu}=\frac{1}{2} \mathcal{F}_{a b} \mathrm{~d} x^{a} \wedge \mathrm{d} x^{b}+\mathcal{F}_{a 3} \mathrm{~d} x^{a} \wedge \mathrm{d} x^{3} .
\end{gathered}
$$

On $M^{4}=\Sigma_{3} \times S^{1}$, with its boundary $\partial M^{4}=\partial \Sigma_{3} \times S^{1}=\Sigma_{2} \times S^{1}$, the (super)group of gauge transformations is naturally defined as (see e.g., Refs. 21 and 22)

$$
\mathcal{G}=\left\{g: M^{4} \rightarrow G \mid g_{\mid \partial M^{4}}=\mathrm{Id}\right\} .
$$

This corresponds to a framing of the gauge bundle over the boundary. For closed membranes, we keep the framing over $S^{1}$.

Employing the adiabatic approach, ${ }^{10,11,15,16,22,23}$ we deform the metric (5),

$$
\mathrm{d} s_{\varepsilon}^{2}=g_{\mu \nu}^{\varepsilon} \mathrm{d} x^{\mu} \mathrm{d} x^{\nu}=g_{a b} \mathrm{~d} x^{a} \mathrm{~d} x^{b}+\varepsilon^{2}\left(\mathrm{~d} x^{3}\right)^{2},
$$


where $\varepsilon \in[0, \infty)$ is a real parameter. This is equivalent to scaling the radius of our circle, replacing it with $S_{\varepsilon}^{1}$ of radius $\varepsilon$. Indices are raised by $g_{\varepsilon}^{\mu \nu}$, and we have

$$
\mathcal{F}_{\varepsilon}^{a b}=g_{\varepsilon}^{a c} g_{\varepsilon}^{b d} \mathcal{F}_{c d}=\mathcal{F}^{a b} \quad \text { and } \quad \mathcal{F}_{\varepsilon}^{a 3}=g_{\varepsilon}^{a c} g_{\varepsilon}^{33} \mathcal{F}_{c 3}=\varepsilon^{-2} \mathcal{F}^{a 3},
$$

where indices in $\mathcal{F}^{\mu \nu}$ have been raised by the non-deformed metric tensor components $g^{\mu \nu}$. In addition, we have $\operatorname{det}\left(g_{\mu \nu}^{\varepsilon}\right)=\varepsilon^{2} \operatorname{det}\left(g_{\mu \nu}\right)$.

We consider the Yang-Mills action functional with a cosmological constant $\Lambda$ of the form

$$
S_{\varepsilon}=\int_{M^{4}} \mathrm{~d}^{4} x \sqrt{\left|\operatorname{det} g_{\Sigma_{3}}\right|}\left\{\frac{\varepsilon^{2}}{2}\left\langle\mathcal{F}_{a b} \mathcal{F}^{a b}\right\rangle+\left\langle\mathcal{F}_{a 3} \mathcal{F}^{a 3}\right\rangle+\Lambda\right\} .
$$

For $\varepsilon=1$ and $\Lambda=0$, it coincides with the standard Yang-Mills action. The value of $\Lambda$ will be fixed later.

\section{EULER-LAGRANGE EQUATIONS}

For the deformed metric, the Yang-Mills equations take the form

$$
\begin{aligned}
\varepsilon^{2} D_{a} \mathcal{F}^{a b}+D_{3} \mathcal{F}^{3 b} & =0 \\
\text { and } \quad D_{a} \mathcal{F}^{a 3} & =0 .
\end{aligned}
$$

Allowing also the metric $g_{\Sigma_{3}}$ on $\Sigma_{3}$ to vary, its Euler-Lagrange equations give the energy-momentum constraint

$$
T_{a b}^{\varepsilon}=\varepsilon^{2}\left(g^{c d}\left\langle\mathcal{F}_{a c} \mathcal{F}_{b d}\right\rangle-\frac{1}{4} g_{a b}\left\langle\mathcal{F}_{c d} \mathcal{F}^{c d}\right\rangle\right)+\left\langle\mathcal{F}_{a 3} \mathcal{F}_{b 3}\right\rangle-\frac{1}{2} g_{a b}\left(\left\langle\mathcal{F}_{c 3} \mathcal{F}^{c 3}\right\rangle+\Lambda\right)=0
$$

In the adiabatic limit $\varepsilon \rightarrow 0$, our Equations (12)-(14) become

$$
\begin{gathered}
D_{3} \mathcal{F}^{3 b} \equiv \partial_{3} \mathcal{F}^{3 b}+\left[\mathcal{A}_{3}, \mathcal{F}^{3 b}\right]=0, \\
D_{a} \mathcal{F}^{a 3} \equiv \sqrt{\left|\operatorname{det} g_{\Sigma_{3}}\right|^{-1}} \partial_{a}\left(\sqrt{\left|\operatorname{det} g_{\Sigma_{3}}\right|} g^{a b} \mathcal{F}_{b 3}\right)+\left[\mathcal{A}_{a}, \mathcal{F}^{a 3}\right]=0, \\
T_{a b}^{0} \equiv\left\langle\mathcal{F}_{a 3} \mathcal{F}_{b 3}\right\rangle-\frac{1}{2} g_{a b}\left(\left\langle\mathcal{F}_{c 3} \mathcal{F}^{c 3}\right\rangle+\Lambda\right)=0 .
\end{gathered}
$$

\section{MODULI SPACE}

Let us recall how one considers the reduction of Yang-Mills theory from $\mathbb{R}^{3} \times S_{\varepsilon}^{1}$ to $\mathbb{R}^{3}$ while $S_{\varepsilon}^{1}$ shrinks to a point for an ordinary compact Lie group $G .{ }^{13,14,28}$ First, one keeps in lagrangian (11) only the zero modes $\mathcal{A}_{3}^{0}$ in the Fourier expansion on $S_{\varepsilon}^{1}$, which are nothing but the Wilson lines, whose moduli are parametrized by coordinates $\phi^{\alpha}$ of the maximal torus in $G$. These moduli produce a term $\mathcal{F}_{a 3} \mathcal{F}^{a 3}=\delta_{\alpha \beta} \partial_{a} \phi^{\alpha} \partial^{a} \phi^{\beta}$ in the lagrangian. Second, for $\mathcal{F}_{a b}$ smoothly depending on $\varepsilon$, the first term in lagrangian (11) vanishes. However, it was observed ${ }^{13,14}$ that for Dirac monopoles, the components $\mathcal{F}_{a b}$ are related with the magnetic photon, having only one component $\tilde{\mathcal{A}}_{3}^{0}$ along $S_{\varepsilon}^{1}$, via

$$
\varepsilon_{a b c} \mathcal{F}^{b c}=\varepsilon^{-1} \partial_{a} \tilde{\mathcal{A}}_{3}^{0},
$$

where the $\varepsilon^{-1}$ appears from the metric dependence of the Hodge star operator. These monopole configurations correspond to 't Hooft lines around the circle $S_{\varepsilon}^{1}$. They survive in the limit $\varepsilon \rightarrow 0$, yielding in lagrangian (11) an additional term proportional to $\delta_{\alpha \beta} \partial_{a} \psi^{\alpha} \partial^{a} \psi^{\beta}$, where $\psi^{\alpha}$ are coordinates on the Cartan torus in the dual group $G^{\vee}$.

In our case, the situation is different since our supermembrane moves in a noncompact superspace, namely, $G=\operatorname{SUSY}(N=1) / \operatorname{SO}(10,1)$. For any fixed $x^{a} \in \Sigma_{3}$, a generic framed $\mathcal{A}_{3}$ is parametrized by the moduli space

$$
\Omega G=\operatorname{Map}\left(S_{\varepsilon}^{1}, G\right) / G=L G / G,
$$

i.e., the based loop group, and it can be written in the form

$$
\mathcal{A}_{3}=\hat{h}^{-1} \partial_{3} \hat{h}=h^{-1} \mathcal{A}_{3}^{0} h+h^{-1} \partial_{3} h \quad \text { with } \quad \hat{h}=h_{0} h \in \Omega G \quad \text { and } \quad \mathcal{A}_{3}^{0}=h_{0}^{-1} \partial_{3} h_{0} \in \mathfrak{g},
$$


where $h \in \Omega G$ and $h_{0} \in G \subset \Omega G$. Note that neither $\hat{h}$ nor $h$ belong to the gauge group. In fact, (20) defines a map $\hat{h} \mapsto h_{0}$ from $\Omega G$ to $G$. The Wilson lines $\mathcal{A}_{3}^{0}$ are parametrized by $G$. Since our aim is the supermembrane moving in $G$, we choose the magnetic photon component $\tilde{\mathcal{A}}_{3}^{0}$ to vanish. Furthermore, in the spirit of the adiabatic approach, it is assumed that all moduli of $\mathcal{A}_{3}$ are functions of $x^{a} \in \Sigma_{3}$, i.e., both functions $h$ and $h_{0}$ depend on $x^{a}$ via their moduli. We denote by $\mathcal{N}$ the space of all $\mathcal{A}_{3}$ given by (20), and we define the projection $\pi: \mathcal{N} \rightarrow G$ since we want to keep only $\mathcal{A}_{3}^{0}$ in the limit $\varepsilon \rightarrow 0$.

\section{EFFECTIVE ACTION}

The variable $\mathcal{A}_{3}^{0}$, as introduced in (20), depends on $x^{a} \in \Sigma_{3}$ only via the moduli parameters $\left(X^{\alpha}, \theta^{A}\right) \in G$. Then the moduli of $\mathcal{A}_{3}^{0}$ define a map

$$
(X, \theta): \Sigma_{3} \rightarrow G \quad \text { with } \quad\left(X\left(x^{a}\right), \theta\left(x^{a}\right)\right)=\left(X^{\alpha}\left(x^{a}\right), \theta^{A}\left(x^{a}\right)\right) .
$$

The map (21) is not arbitrary, it is constrained by Equations (15)-(17). The derivative $\partial_{a} \mathcal{A}_{3}$ belongs to the tangent space $T_{\mathcal{A}_{3}} \mathcal{N}$. With the help of the projection $\pi: \mathcal{N} \rightarrow G$ with fibres $Q$, one can decompose $\partial_{a} \mathcal{A}_{3}$ into two parts,

$$
T_{\mathcal{A}_{3}} \mathcal{N}=\pi^{*} T_{\mathcal{F}_{3}^{0}} G \oplus T_{\mathcal{A}_{3}} Q \quad \Leftrightarrow \quad \partial_{a} \mathcal{A}_{3}=\Pi_{a}^{\Delta} \xi_{\Delta 3}+D_{3} \epsilon_{a},
$$

where $\Delta=(\alpha, A)$ and

$$
\Pi_{a}^{\alpha}=\partial_{a} X^{\alpha}-\mathrm{i} \bar{\theta} \gamma^{\alpha} \partial_{a} \theta \quad \text { and } \quad \Pi_{a}^{A}=\partial_{a} \theta^{A} .
$$

In (22), $\epsilon_{a}$ are g-valued parameters $\left(D_{3} \epsilon_{a} \in T_{\mathcal{A}_{3}} Q\right)$, and the vector fields $\xi_{\Delta 3}$ on $G$ can be identified with the generators $\xi_{\Delta}=\left(\xi_{\alpha}, \xi_{A}\right)$ of $G$.

On $\xi_{\Delta 3}$, we impose the gauge-fixing condition

$$
D_{3} \xi_{\Delta 3}=0 \stackrel{(22)}{\Longrightarrow} D_{3} D_{3} \epsilon_{a}=D_{3} \partial_{a} \mathcal{A}_{3} .
$$

Recall that $\mathcal{A}_{3}$ is determined by (20) and $\mathcal{A}_{a}$ are yet free. In the adiabatic approach, one can naturally choose $\mathcal{A}_{a}=\epsilon_{a}$ (cf., Refs. 15 and 23), where $\epsilon_{a}$ are defined from (24). Then one obtains

$$
\mathcal{F}_{a 3}=\partial_{a} \mathcal{A}_{3}-D_{3} \mathcal{A}_{a}=\partial_{a} \mathcal{A}_{3}-D_{3} \epsilon_{a}=\Pi_{a}^{\Delta} \xi_{\Delta 3} \in T_{\mathcal{A}_{3}^{0}} G
$$

Substituting (25) into (15), we see that the latter is resolved due to (24). Plugging (25) into the action (11) with $\varepsilon \rightarrow 0$ and fixing $\Lambda=-1$, we obtain the effective action

$$
S_{0}=2 \pi \int_{\Sigma_{3}} \mathrm{~d}^{3} x \sqrt{\left|\operatorname{det} g_{\Sigma_{3}}\right|}\left(g^{a b} \Pi_{a}^{\alpha} \Pi_{b}^{\beta} \eta_{\alpha \beta}-1\right) .
$$

It coincides with the kinetic part of the supermembrane action. ${ }^{5}$ One may also show (cf., Ref. 19) that Equation (16) are equivalent to the Euler-Lagrange equations for $\left(X^{\alpha}, \theta^{A}\right)$ following from (26). Finally, substituting (25) into (17), we arrive at

$$
\Pi_{a}^{\alpha} \Pi_{b}^{\beta} \eta_{\alpha \beta}-\frac{1}{2} g_{a b}\left(g^{c d} \Pi_{c}^{\alpha} \Pi_{d}^{\beta} \eta_{\alpha \beta}-1\right)=0
$$

which may also be obtained from (26) by varying the metric.

From (27), it follows that

$$
g_{a b}=\eta_{\alpha \beta} \Pi_{a}^{\alpha} \Pi_{b}^{\beta},
$$

and, after putting this back into (26), we get the standard Nambu-Goto lagrangian for the supermembrane. It is obvious that for $\theta=0$, the bosonic membrane action remains.

\section{WESS-ZUMINO-TYPE TERM}

The action (26) is not the full supermembrane action, since the latter needs also a WessZumino-type term. ${ }^{5,7}$ Continuing our "reverse engineering" strategy, we look for an addition to the 
Yang-Mills action (11) which in the infrared limit $\varepsilon \rightarrow 0$ will give us this Wess-Zumino-type term. This addition can be incorporated as follows. We extend $\Sigma_{3}$ to a Lorentzian 4-manifold $\Sigma_{4}$ with boundary $\Sigma_{3}=\partial \Sigma_{4}$ and (local) coordinates $x^{\hat{a}}, \hat{a}=0,1,2,4$. On $\Sigma_{4}$, one introduces the four-form ${ }^{5,7}$

$\Omega_{4}=\langle\Pi \wedge \Pi \wedge \Pi \wedge \Pi\rangle=f_{\Delta \Lambda \Sigma \Gamma} \Pi^{\Delta} \wedge \Pi^{\Lambda} \wedge \Pi^{\Sigma} \wedge \Pi^{\Gamma}=\hat{\mathrm{d}} \bar{\theta} \gamma_{[\alpha} \gamma_{\beta]} \wedge \hat{\mathrm{d}} \theta \wedge \Pi^{\alpha} \wedge \Pi^{\beta}=\hat{\mathrm{d}} \Omega_{3}$

for $\Pi:=\Pi_{\hat{a}} \mathrm{~d} x^{\hat{a}}=\Pi_{\hat{a}}^{\Delta} \mathrm{d} x^{\hat{a}} \xi_{\Delta}$, where $\hat{\mathrm{d}}=\mathrm{d} x^{\hat{a}} \partial_{\hat{a}}$. The explicit form of the constants $f_{\Delta \Lambda \Sigma \Gamma}$ and the three-form $\Omega_{3}$ can be found in Refs. 5 and 7. Then one adds to the action (26) the term

$$
S_{W Z}=\int_{\Sigma_{4}} \Omega_{4}=\int_{\Sigma_{3}} \Omega_{3},
$$

which completes the M2-brane action. In the setup we investigate here, we take the direct product manifold $\Sigma_{4} \times S^{1}$, extend the index $a$ in (23) to $\hat{a}=0,1,2,4$ and introduce one-forms on $\Sigma_{4}$,

$$
F_{3}:=\mathcal{F}_{\hat{a} 3} \mathrm{~d} x^{\hat{a}} .
$$

Adding (with a proper coefficient) the Wess-Zumino-type term

$$
S_{W Z}^{Y M}=\int_{\Sigma_{4} \times S^{1}} f_{\Delta \Lambda \Sigma \Gamma} F_{3}^{\Delta} \wedge F_{3}^{\Lambda} \wedge F_{3}^{\Sigma} \wedge F_{3}^{\Gamma} \wedge \mathrm{d} x^{3}
$$

to the action functional $S_{\varepsilon}$ from (11) with $\Lambda=-1$, we obtain the gauge-field action which in the adiabatic limit $\varepsilon \rightarrow 0$ becomes the M2-brane action. This implies that features of Yang-Mills theory with the action $(11)+(32)$ for $\varepsilon \neq 0$ can be reduced to properties of supermembranes by taking the limit $\varepsilon \rightarrow 0$.

\section{ACKNOWLEDGMENTS}

This work was partially supported by the Deutsche Forschungsgemeinschaft Grant No. LE $838 / 13$.

${ }^{1}$ P. A. M. Dirac, “An extensible model of the electron,” Proc. R. Soc. London, Ser. A 268, 57 (1962).

2 P. S. Howe and R. W. Tucker, "A locally supersymmetric and reparametrization invariant action for a spinning membrane," J. Phys. A 10, L155 (1977).

${ }^{3}$ J. Hoppe, "Quantum theory of a massless relativistic surface and a two-dimensional bound state problem," Ph.D. thesis, MIT, Cambridge, MA, USA, 1982.

${ }^{4}$ J. Hughes, J. Liu, and J. Polchinski, "Supermembranes," Phys. Lett. B 180, 370 (1986).

${ }^{5}$ E. Bergshoeff, E. Sezgin, and P. K. Townsend, "Supermembranes and eleven-dimensional supergravity," Phys. Lett. B 189, 75 (1987)

${ }^{6}$ B. de Wit, J. Hoppe, and H. Nicolai, “On the quantum mechanics of supermembranes,” Nucl. Phys. B 305, 545 (1988).

7 J. A. de Azcarraga and P. K. Townsend, "Superspace geometry and classification of supersymmetric extended objects," Phys. Rev. Lett. 62, 2579 (1989).

8 J. Hoppe, "Relativistic membranes," J. Phys. A 46, 023001 (2013).

9 J. Bagger, N. Lambert, S. Mukhi, and C. Papageorgakis, "Multiple membranes in M-theory," Phys. Rept. 527, 1 (2013); e-print arXiv:1203.3546 [hep-th].

10 S. Dostoglou and D. A. Salamon, "Self-dual instantons and holomorphic curves," Ann. Math. 139, 581 (1994).

${ }^{11}$ S. K. Donaldson and R. P. Thomas, "Gauge theory in higher dimensions," in The Geometric Universe (Oxford University Press, Oxford, 1998)

12 J. A. Harvey, G. W. Moore, and A. Strominger, "Reducing S-duality to T-duality," Phys. Rev. D 52, 7161 (1995); e-print arXiv:hep-th/9501022.

${ }^{13}$ N. Seiberg and E. Witten, "Gauge dynamics and compactification to three-dimensions," in The Mathematical Beauty of Physics: Saclay (World Scientific, Singapore, 1997), pp. 333-366, e-print arXiv:hep-th/9607163.

14 N. Seiberg, "Notes on theories with 16 supercharges," Nucl. Phys. Proc. Suppl. 67, 158 (1998); e-print arXiv:hep-th/ 9705117.

15 N. S. Manton, “A remark on the scattering of BPS monopoles,” Phys. Lett. B 110, 54 (1982).

${ }^{16}$ D. Stuart, "The geodesic approximation for the Yang-Mills-Higgs equations," Commun. Math. Phys. 166, 149 (1994).

17 G. Tian, "Gauge theory and calibrated geometry," Ann. Math. 151, 193 (2000); e-print arXiv:math/0010015 [math-dg].

18 A. Deser, O. Lechtenfeld, and A. D. Popov, "Sigma-model limit of Yang-Mills instantons in higher dimensions," Nucl. Phys. B 894, 361 (2015); e-print arXiv:1412.4258 [hep-th].

19 A. D. Popov, "String theories as the adiabatic limit of Yang-Mills theory," Phys. Rev. D 92, 045003 (2015); e-print arXiv: 1505.07733 [hep-th].

${ }^{20}$ A. D. Popov, "Green-Schwarz superstring as subsector of Yang-Mills theory," e-print arXiv:1506.02175 [hep-th] (unpublished).

${ }^{21}$ S. K. Donaldson, "Boundary value problems for Yang-Mills fields," J. Geom. Phys. 8, 89 (1992). 
22 D. A. Salamon, "Notes on flat connections and the loop group," Preprint, University of Warwick, 1998.

${ }^{23}$ E. J. Weinberg and P. Yi, "Magnetic monopole dynamics, supersymmetry, and duality," Phys. Rept. 438, 65 (2007); e-print arXiv:hep-th/0609055.

${ }^{24}$ See Refs. 8 and 9 for historical reviews and more references.

25 The direct product structure is not necessary for the application of the adiabatic method. In general, it is enough if there is a fibration $Z \rightarrow X$ or if $X$ is a calibrated submanifold of $Z$.

${ }^{26}$ In the physics literature this limit is called infrared or low-energy limit (see e.g., Refs. 12-14).

${ }^{27}$ In fact, in Refs. $12-14$ the authors considered $\mathcal{N}=4$ and $\mathcal{N}=2$ super-Yang-Mills theories but the restriction to the pure Yang-Mills subsector does not change the picture.

${ }^{28}$ For simplicity, we restrict ourselves to the pure Yang-Mills subsector of the supersymmetric theories in Refs. 13 and 14. 